\title{
Review Article \\ Effects of Calcium Ion, Calpains, and Calcium Channel Blockers on Retinitis Pigmentosa
}

\author{
Mitsuru Nakazawa \\ Department of Ophthalmology, Hirosaki University Graduate School of Medicine, 5 Zaifu-cho, Hirosaki 036-8561, Japan \\ Correspondence should be addressed to Mitsuru Nakazawa, mitsuru@cc.hirosaki-u.ac.jp \\ Received 26 June 2010; Revised 13 September 2010; Accepted 15 November 2010 \\ Academic Editor: Radha Ayyagari
}

Copyright ( 2011 Mitsuru Nakazawa. This is an open access article distributed under the Creative Commons Attribution License, which permits unrestricted use, distribution, and reproduction in any medium, provided the original work is properly cited.

Recent advances in molecular genetic studies have revealed many of the causative genes of retinitis pigmentosa (RP). These achievements have provided clues to the mechanisms of photoreceptor degeneration in RP. Apoptosis is known to be a final common pathway in RP and, therefore, a possible therapeutic target for photoreceptor rescue. However, apoptosis is not a single molecular cascade, but consists of many different reactions such as caspase-dependent and caspase-independent pathways commonly leading to DNA fractionation and cell death. The intracellular concentration of calcium ions is also known to increase in apoptosis. These findings suggest that calpains, one of the calcium-dependent proteinases, play some roles in the process of photoreceptor apoptosis and that calcium channel antagonists may potentially inhibit photoreceptor apoptosis. Herein, the effects of calpains and calcium channel antagonists on photoreceptor degeneration are reviewed.

\section{Introduction}

Retinitis pigmentosa (RP) represents a group of hereditary retinal degenerations principally characterized by progressive rod-dominant photoreceptor degeneration in the initial stage and eventual cone photoreceptor degeneration in later stages. Patients with RP mainly complain of night blindness and photophobia in the early stage, followed by gradual constriction of the visual field, decreased visual acuity, and color blindness in later stages. The prevalence of RP is roughly 1 in 4,000-5,000 people, and the condition is common in both Asian and Western countries. Significant features of RP include heterogeneity in both clinical and genetic characteristics. For instance, the severity and progression of RP vary from patient to patient even in the same family, despite affected members presumably sharing the same causative gene mutation. Heredities are also heterogeneous, characterized by at least 3 different modes of inheritance, such as autosomaldominant, autosomal-recessive, and X-linked patterns. Since a mutation in the rhodopsin gene was first identified as causing one type of autosomal-dominant RP [1], at least 48 different causative genes have been identified (RetNet: http://www.sph.uth.tmc.edu/retnet/disease.htm); however, many other putative causative genes and mutations have yet to be identified. Molecular genetic studies have also demonstrated that a primary lesion in RP involves photoreceptor and/or retinal pigment epithelial cells in which many causative genes are specifically expressed under physiological conditions. Photoreceptor or retinal pigment epithelial cells are known to degenerate mostly through apoptosis [2], which is now understood as a final common pathway for $\mathrm{RP}$ at the cellular level. As the mechanisms of photoreceptor degeneration have been gradually elucidated, studies on therapeutic approaches have dramatically increased, including pharmacotherapy, cellular transplantation, gene therapy, regenerative therapy, and retinal prosthesis. This paper mainly focuses on studies examining the effects of calcium ions and calpains on photoreceptor apoptosis, as well as pharmacological treatments for RP using calcium channel antagonists.

\section{Genetic Background of RP}

One of the most important breakthroughs in RP research was the identification of a point mutation $(\mathrm{P} 23 \mathrm{H})$ in the rhodopsin gene as a causative gene mutation for one form of autosomal-dominant RP $[1,3]$. Since then, using a candidate gene approach, various mutations in the rhodopsin gene and 
many other genes have been identified in several RP families. These include mutations in the genes encoding $\beta$ - and $\alpha$ subunits of rod cGMP-phosphodiesterase for autosomalrecessive $\mathrm{RP}[4,5]$ and peripherin/RDS (RDS: retinal degeneration slow) for autosomal-dominant RP $[6,7]$. These findings in the early 1990s suggested to many researchers that RP is caused by a single or one allelic pair of mutations in one of the genes specifically or dominantly expressed in photoreceptor cells. The candidate gene approach was also relatively easy to perform once researchers suspected genes already known to be retina specific as possible candidates for RP. Many other genes and mutations in these genes were then found to cause RP (Table 1). However, the candidate gene approach is limited in that screening can only be performed for known genes and involvement could not be ascertained for previously unknown genes that might be expressed not only in the retina, but also in other organs or tissues in a ubiquitous fashion. For these reasons, genetic linkage and/or association analyses have been performed in combination with a candidate gene approach to identify many other previously unpredictable genes as causative genes for RP. This group includes PRPF31 [8], PRPF3 [9], PRPF8 [10], IMPDH1 [11], Mertk [12, 13], and CA4 [14] which are expressed in other tissues besides retina (Table 1). These findings indicate that photoreceptors and retinal pigment epithelium are much more active in protein synthesis than any other tissues and show high levels of gene expression and protein metabolism. In addition, molecular genetic studies have disclosed that RP is genetically more heterogeneous than it used to be considered and that the genetic heterogeneity may be one explanation for the clinical heterogeneity.

\section{Photoreceptor Apoptosis as a Common Mechanism in RP}

Despite the clinical and genetic heterogeneity, RP demonstrates common features derived from rod-predominant degeneration. This essential phenomenon allowed researchers to suspect some common mechanisms leading to photoreceptor cell death once the patient carries a single or one allelic pair of many causative gene mutations. Apoptosis is a genetically programmed mechanism that leads cells to death, and RP has been known to be initiated by photoreceptor apoptosis as a final common pathway at the cellular level, irrespective of gene mutations. For instance, apoptosis was detected in retinal degeneration 1 (rd1), rds, and rhodopsin mutant mice [2]. To date, many pathways have been found for apoptosis itself, involving caspases, cathepsins, calpains, apoptosis-inducing factor (AIF), Fas, and more. Once abnormal and/or insufficient structural or metabolic stresses induced by a certain gene mutation exceed predetermined thresholds that a cell can tolerate, mechanisms of apoptosis are initiated that lead to nuclear DNA fragmentation and subsequent cell death. Many experimental studies have supported that caspasedependent or -independent apoptotic pathways are activated during experimental retinal degeneration models $[15,16]$.
TABLE 1: List of causative genes of RP: retina specific and nonspecific.

\begin{tabular}{|c|c|c|c|}
\hline Category & $\operatorname{ADRP}(20)$ & $\operatorname{ARRP}(25)$ & XLRP (2) \\
\hline \multirow[t]{24}{*}{ Retina specific } & CRX & ABCA4 & RP2 \\
\hline & FSCN2 & CERKL & \\
\hline & GUCA1B & CNGA1 & \\
\hline & NRL & CNGB1 & \\
\hline & NR2E3 & CRB1 & \\
\hline & PRPH2 & EYES & \\
\hline & RDH12 & IDH3B & \\
\hline & $\mathrm{RHO}$ & LRAT & \\
\hline & ROM1 & NR2E3 & \\
\hline & RP1 & NRL & \\
\hline & RP9 & PDE6A & \\
\hline & SEMA4A & PDE6B & \\
\hline & & PRCD & \\
\hline & & PROM1 & \\
\hline & & $\mathrm{RBP} 3$ & \\
\hline & & RGR & \\
\hline & & RHO & \\
\hline & & RLBP1 & \\
\hline & & $\mathrm{RP} 1$ & \\
\hline & & RPE65 & \\
\hline & & SAG & \\
\hline & & SPATA7 & \\
\hline & & TUP1 & \\
\hline & & USH2 & \\
\hline \multirow[t]{8}{*}{ Retina nonspecific } & CA4 & MERTK & RPGR \\
\hline & IMPDH1 & & \\
\hline & KHLH7 & & \\
\hline & PRPF3 & & \\
\hline & PRPF8 & & \\
\hline & PRPF31 & & \\
\hline & SNRNP200 & & \\
\hline & TOPORS & & \\
\hline
\end{tabular}

Abbreviations are listed in Ret:Net: http://www.sph.uth.tmc.edu/retnet/ disease.htm.

Apoptosis can thus be considered as a therapeutic target as it plays many roles in retinitis pigmentosa $[17,18]$.

Calpains [EC 3.4.22.17], a group of calcium-dependent cysteine proteases, play some important roles in caspaseindependent photoreceptor apoptotic pathways with lightinduced retinal damage [19] and in rd1 mice [20, 21] and Royal College of Surgeons (RCS) rats [22] as models of retinal degeneration. Calpains are also involved in calciuminduced cell death in a murine photoreceptor-derived cell line $[23,24]$. There is little doubt that intracellular concentrations of calcium ion were elevated in apoptosis [2528]. As calcium influx is actually elevated in degenerating rd1 rod photoreceptors $[20,29]$, calpains are suspected to play important roles in photoreceptor apoptosis in RP. In addition, calpain inhibitors and calcium channel blockers appear to offer reasonable candidates at least in part as 
pharmacotherapeutic agents for RP. Transient inhibitory effects of calpain inhibitors on photoreceptor apoptosis in RCS rats have recently been described by Mizukoshi et al. [22].

\section{Effects of Calcium Ion on Photoreceptor Apoptosis}

As mentioned above, intracellular concentrations of calcium ion are increased in apoptosis [20, 25-29]. Intracellular calcium ions are provided through several types of calcium channels and transporters located on cell membranes, endoplasmic reticulum, and mitochondria. Cyclic-nucleotidegated cation channels (CNGCs) are located in the outer segment and closed by depletion of cGMP as a result of the phototransduction reaction triggering hyperpolarization. Ltype voltage-gated calcium channels (VGCCs) are located in the cell body and synaptic terminal and are closed by hyperpolarization of the cell membrane induced by phototransduction. Steele Jr. et al. [30] suggested that the average concentration of calcium in the terminal ranges from $\sim 350 \mathrm{nM}$ in hyperpolarized light-adapted cells to more than $39 \mu \mathrm{M}$ in cells depolarized to dark potentials in salamander rods and cones. In addition to CNGC and VGCC, intracellular concentrations of calcium ions are regulated by many other factors, such as plasma membrane calcium ATPase, store-operated calcium entry, calcium stores in the endoplasmic reticulum, and mitochondria (Figure 1). Under pathological conditions, like those in $\mathrm{rd} 1 \mathrm{mice}$, intracellular calcium levels significantly increase in rods, even before the detection of apoptotic cells [29]. The marked elevation of intracellular concentrations of calcium ions activates downstream reactions, including hydrolytic enzymes like calpains, and eventually leads to cell death [25]. Excessive calcium influx is initiated in the cytosol and subsequently in mitochondria in rd1 mouse [29], suggesting that increased calcium ions may affect many biochemical cascades and reactions not only in the cytosol but also in the mitochondria $[31,32]$. As mentioned above, increased intracellular calcium concentrations activate calpains, leading to the activation of both caspase-dependent and -independent apoptotic pathways. First, as a caspase-dependent pathway, calpains activate caspase 12 , which sequentially activates caspases 9 , 3 , 4, and 7 and finally apoptosis is upregulated. Second, cytosolic calpains further activate cathepsins and mitochondrial calpains activate AIF, which subsequently translocates from mitochondria to the nucleus [22]. This reaction has been speculated to represent one of the caspase-independent pathways of apoptosis $[33,34]$.

\section{5. $\mathrm{Ca}^{2+}$ Channel Antagonists for Photoreceptor Apoptosis in Animal Experiments}

Since Frasson et al. [35] first reported the effects of Dcis-diltiazem, a benzothiazepin calcium channel antagonist which blocks both CNGC and VGCC, on photoreceptor protection in $\mathrm{rd} 1$ mice, several investigators have reported positive and negative effects of calcium channel blockers on animal models of RP [19-21, 36-43]. Since rd1 is caused by a mutation in the gene encoding the $\beta$-subunit of rod cGMP-phosphodiesterase, one of the key enzymes in the phototransduction pathway, CNGCs located in the outer segment cannot be closed despite light stimulation in the rod photoreceptor cells. Inhibition of light-induced hyperpolarization, caused by a mutation in the rod cGMPphosphodiesterase gene, also does not close VGCC. These phenomena increase calcium influx in both outer and inner segments in $\mathrm{rd} 1 \mathrm{mice}$. The intracellular concentration of calcium ions is subsequently elevated, leading to photoreceptor apoptosis [35], possibly by upregulation of calpains and other proteins [28]. Sanges et al. [20] demonstrated that systemic administration of D-cis-diltiazem reduced intracellular concentrations of calcium, downregulating calpains and photoreceptor apoptosis in $\mathrm{rd} 1 \mathrm{mice}$. Direct inhibitory effects of D-cis-diltiazem on CNGC [44] or L-type VGCC [39] have been reported, and D-cis-diltiazem effectively blocks photoreceptor light damage in mouse models by inhibiting photoreceptor apoptosis [19]. In contrast, L-cis isomer inhibits L-type VGCC similarly to D-cis isomer [45]. The difference in action between D-cis- and L-cisdiltiazems on photoreceptor neuroprotection [35] suggests that CNGC might also be important for photoreceptor neuroprotection [44]. Read et al. [46] also reported that the $\beta$-subunit of VGCC knock-out rd1 mice showed retardation of photoreceptor degeneration, suggesting that blockage of calcium influx may partially contribute to photoreceptor rescue in these animal models although it did not prevent photoreceptor degeneration. Despite these studies, however, Pawlyk et al. [36] and Takano et al. [41] found no rescue effects of D-cis-diltiazem on retinal degeneration in $\mathrm{rd} 1$ mice, and Bush et al. [42] also reported that D-cis-diltiazem was ineffective for photoreceptor rescue in rhodopsin $\mathrm{P} 23 \mathrm{H}$ transgenic rats.

While the effects of diltiazem on animal models of retinal degeneration remain controversial, another type of calcium channel blocker, nilvadipine, a member of the dihydropyridine derivatives, is another candidate therapeutic agent for RP. Nilvadipine has low-voltage-activated calcium blocking actions in addition to L-type high-voltage calcium blocking actions. The hydrophobic nature induced by the chemical structure of nilvadipine allows high permeability to the central nervous system, including the retina [47]. Systemic administration of nilvadipine has been shown to be effective for protecting photoreceptors in RCS rats [37, 40], rd1 mice [41], and heterozygous $\mathrm{rd} 2$ ( $\mathrm{rds}$ ) mice [43]. In addition to direct effects of calcium channel blockers on intracellular concentrations of calcium ion in photoreceptor cells, other indirect effects are expected such as increased expression of fibroblast growth factor (FGF) 2 [40, 41] and ciliary neurotrophic factor (CNTF) [43] in the retina, and increased choroidal blood flow [48]. Since FGF2 and CNTF are known to exert photoreceptor-protective effects [49-56], upregulating such intrinsic neurotrophic factors by nilvadipine may demonstrate beneficial effects against RP. CNTF has also been applied as a clinical trial for RP [57]. In addition, oxidative stress may be involved in photoreceptor death in RP [58-63], and nilvadipine has 


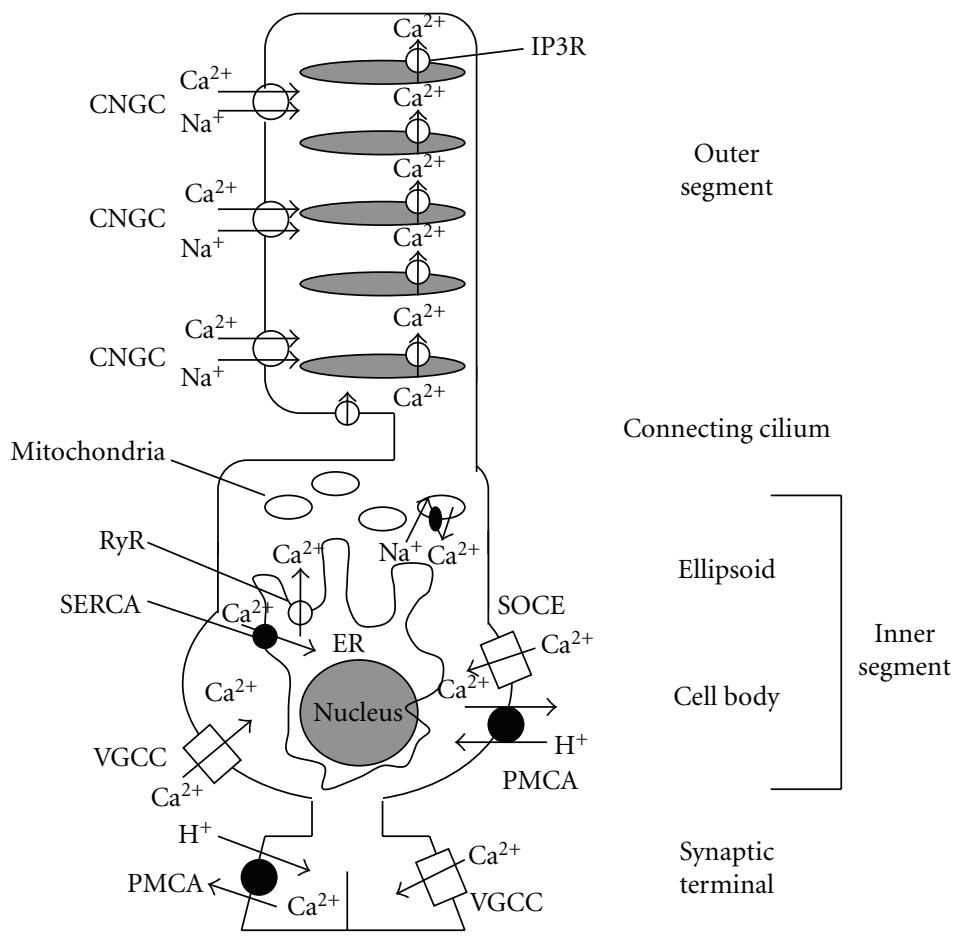

FIGURE 1: Schematic view of calcium channels and transporters in the rod-photoreceptor. Abbreviations: CNGC, cyclic-nucleotide-gated cation channel; VGCC: voltage-gated calcium channel; PMCA: plasma membrane calcium ATPase; SOCE: store-operated calcium entry; ER: endoplasmic reticulum; SERCA: sarcoplasmic-endoplasmic reticulum Ca ${ }^{2+}$-ATPase; IP3R: inositol 1, 4, 5-triphoaphate receptor; RyR: ryanodine receptor. $\mathrm{Ca}^{2+}$ stock and release from the mitochondria are mediated by $\mathrm{Ca}^{2+}$ uniporter channels and $\mathrm{Na}^{+} / \mathrm{Ca}^{2+}$ transporters.

the highest antioxidant potency among calcium channel blockers [64]. The direct effects of calcium channel blockers on photoreceptor calpains have not yet been studied. Studies involving calcium channel antagonists are listed in Table 2. As the effects of calcium channel blockers on photoreceptor rescue remain controversial, further biochemical studies are required in order to facilitate our understanding of the mechanisms of photoreceptor degeneration induced by various types of gene mutations, the effects of intracellular calcium ions on downstream reactions, and the effects of calcium channel blockers on both concentrations of calcium ions and downstream reactions in various types of heterogeneous conditions of RP. Although human RP is caused by various kinds of heterogeneous causative gene mutations, our understanding regarding photoreceptor degeneration in $\mathrm{RP}$ is still limited to relatively small numbers of experimental models of RP.

\section{Human Trials}

Although human RP is genetically heterogeneous, possible rescue effects of calcium channel blockers on photoreceptor degeneration in certain animal models of RP, such as rd1 and rds mice and RCS rats, have encouraged researchers to expect therapeutic effects of calcium channel blockers for RP. Pasantes-Morales et al. [65] reported that a combination of D-cis-diltiazem, taurin, and vitamin $\mathrm{E}$ has beneficial effects on the visual field progression, although the study did not clarify whether diltiazem alone demonstrated beneficial effects. Ohguro [66] reported the photoreceptor rescue effects of nilvadipine in a small patient group. We expanded his nilvadipine study for RP patients to confirm the results. Although both treated and control groups are still small, our results have shown significant retardation of the mean deviation (MD) slope as calculated by the central visual field (Humphry Visual Field Analyzer, 10-2 Program) after a mean of 48 months of observation [67]. As these pilot studies are small-sized and cannot completely exclude possible biases, a large-scale, randomized, multicenter human trial of calcium channel blockers is required in order to evaluate their efficacy as therapeutic agents for RP.

\section{Future Insights}

As pharmacotherapeutic agents for RP, vitamin A [68, 69] and lutein [70] are reportedly effective in slowing $\mathrm{RP}$, and carbonic anhydrase inhibitors appear effective for reducing chronic cystoid macular edema [71, 72], although the basic molecular mechanisms underlying these actions remain unclear. Effects of calcium channel blockers have been speculated based on the molecular mechanisms in RP identified in recent molecular genetic [4] and animal studies [20,35] of RP and also research on neuroprotection for glaucoma [73]. In addition to previous pilot studies, large-scale human trials to examine the effects of calcium channel blockers in the progression of RP are needed to 
TABLE 2: Photoreceptor rescue by calcium channel antagonists.

\begin{tabular}{|c|c|c|c|}
\hline & Authors & Year & Ref. \\
\hline \multicolumn{4}{|l|}{ Supportive } \\
\hline Diltiazem on rd1 mouse & Frasson et al. & 1999 & {$[35]$} \\
\hline Diltiazem on rd1 mouse & Sanges et al. & 2006 & {$[20]$} \\
\hline Diltiazem on light damage & Vallaza-Deschamps et al. & 2005 & [19] \\
\hline Calcium channel knockout on rd1 & Read et al. & 2002 & {$[46]$} \\
\hline Nilvadipine on rd1 mouse & Takano et al. & 2004 & {$[41]$} \\
\hline Nilvadipine on RCS rat & Yamazaki et al. & 2002 & {$[37]$} \\
\hline Nilvadipine on RCS rat & Sato et al. & 2003 & {$[40]$} \\
\hline Nilvadipine on rd2 (rds) mouse & Takeuchi et al. & 2008 & {$[43]$} \\
\hline \multicolumn{4}{|l|}{ Negative } \\
\hline Diltiazem on rd1 mouse & Pawlyk et al. & 2002 & {$[36]$} \\
\hline Diltiazem on rd1 mouse & Takano et al. & 2004 & {$[41]$} \\
\hline Diltiazem on pde $\beta$ knock-out dog & Pearce-Kelling et al. & 2001 & {$[21]$} \\
\hline Diltiazem on rhodopsin $\mathrm{P} 23 \mathrm{H}$ rat & Bush et al. & 2000 & {$[42]$} \\
\hline
\end{tabular}

obtain solid evidence-based results. Since calcium channel blockers may not effectively block enough calcium influx to rescue degenerating photoreceptors depending on the kinds of gene mutations, downstream reactions like calpains should be considered when planning therapy. Effects of calpain inhibitors on human RP patients should also be examined in the future. As other modern technologies have advanced, new therapeutic modalities including gene therapy, retinal prostheses, and regenerative medicine have become increasingly developed, and some applications of these technologies are now commercially available. Of note is the fact that pharmacotherapeutic agents aimed at photoreceptor rescue can be used in combination with gene therapy and regenerative medicine.

\section{References}

[1] T. P. Dryja, T. L. McGee, E. Reichel et al., "A point mutation of the rhodopsin gene in one form of retinitis pigmentosa," Nature, vol. 343, no. 6256, pp. 364-366, 1990.

[2] G. Q. Chang, Y. Hao, and F. Wong, "Apoptosis: final common pathway of photoreceptor death in $\mathrm{rd}$, rds, and rhodopsin mutant mice," Neuron, vol. 11, no. 4, pp. 595-605, 1993.

[3] P. McWilliams, G. J. Farrar, P. Kenna et al., "Autosomal deminant retinitis pigmentosa (ADRP): location of an ADRP gene to the long arm of chromosome 3," Genomics, vol. 53, pp. 619-622, 1989.

[4] M. E. McLaughlin, M. A. Sandberg, E. L. Berson, and T. P. Dryja, "Recessive mutations in the gene encoding the $\beta$ subunit of rod phosphodiesterase in patients with retinitis pigmentosa," Nature Genetics, vol. 4, no. 2, pp. 130-134, 1993.

[5] T. P. Dryja, D. E. Rucinski, S. H. Chen, and E. L. Berson, "Frequency of mutations in the gene encoding the $\alpha$ subunit of rod cGMP- phosphodiesterase in autosomal recessive retinitis pigmentosa," Investigative Ophthalmology and Visual Science, vol. 40, no. 8, pp. 1859-1865, 1999.

[6] G. J. Farrar, P. Kenna, S. A. Jordan et al., "A three-base-pair deletion in the peripherin-RDS gene in one form of retinitis pigmentosa," Nature, vol. 354, no. 6353, pp. 478-480, 1991.
[7] K. Kajiwara, L. B. Hahn, S. Mukai, G. H. Travis, E. L. Berson, and T. P. Dryja, "Mutations in the human retinal degeneration slow gene in autosomal dominant retinitis pigmentosa," Nature, vol. 354, no. 6353, pp. 480-483, 1991.

[8] E. N. Vithana, L. Abu-Safieh, M. J. Allen et al., "A human homolog of yeast pre-mRNA splicing gene, PRP31, underlies autosomal dominant retinitis pigmentosa on chromosome 19q13.4 (RP11)," Molecular Cell, vol. 8, no. 2, pp. 375-381, 2001.

[9] C. F. Chakarova, M. M. Hims, H. Bolz et al., "Mutations in HPRP3, a third member of pre-mRNA splicing factor genes, implicated in autosomal dominant retinitis pigmentosa," Human Molecular Genetics, vol. 11, no. 1, pp. 87-92, 2002.

[10] A. B. McKie, J. C. McHale, T. J. Keen et al., "Mutations in the pre-mRNA splicing factor gene PRPC8 in autosomal dominant retinitis pigmentosa (RP13)," Human Molecular Genetics, vol. 10, no. 15, pp. 1555-1562, 2001.

[11] S. J. Bowne, L. S. Sullivan, S. H. Blanton et al., "Mutations in the inosine monophosphate dehydrogenase 1 gene (IMPDH1) cause the RP10 form of autosomal dominant retinitis pigmentosa," Human Molecular Genetics, vol. 11, no. 5, pp. 559-568, 2002.

[12] P. M. D’Cruz, D. Yasumura, J. Weir et al., "Mutation of the receptor tyrosine kinase gene Mertk in the retinal dystrophic RCS rat," Human Molecular Genetics, vol. 9, no. 4, pp. 645-651, 2000.

[13] A. Gal, Y. Li, D. A. Thompson et al., "Mutations in MERTK, the human orthologue of the RCS rat retinal dystrophy gene, cause retinitis pigmentosa," Nature Genetics, vol. 26, no. 3, pp. 270-271, 2000.

[14] G. Rebello, R. Ramesar, A. Vorster et al., "Apoptosis-inducing signal sequence mutation in carbonic anhydrase IV identified in patients with the RP17 form of retinitis pigmentosa," Proceedings of the National Academy of Sciences of the United States of America, vol. 101, no. 17, pp. 6617-6622, 2004.

[15] C. Jomary, M. J. Neal, and S. E. Jones, "Characterization of cell death pathways in murine retinal neurodegeneration implicates cytochrome $\mathrm{c}$ release, caspase activation, and Bid cleavage," Molecular and Cellular Neuroscience, vol. 18, no. 4, pp. 335-346, 2001.

[16] F. Doonan, M. Donovan, and T. G. Cotter, "Caspaseindependent photoreceptor apoptosis in mouse models of 
retinal degeneration," Journal of Neuroscience, vol. 23, no. 13, pp. 5723-5731, 2003.

[17] F. Doonan and T. G. Cotter, "Apoptosis: a potential therapeutic target for retinal degenerations," Current Neurovascular Research, vol. 1, no. 1, pp. 41-53, 2004.

[18] S. Cottet and D. F. Schorderet, "Mechanisms of apoptosis in retinitis pigmentosa," Current Molecular Medicine, vol. 9, no. 3, pp. 375-383, 2009.

[19] G. Vallazza-Deschamps, D. Cia, J. Gong et al., "Excessive activation of cyclic nucleotide-gated channels contributes to neuronal degeneration of photoreceptors," European Journal of Neuroscience, vol. 22, no. 5, pp. 1013-1022, 2005.

[20] D. Sanges, A. Comitato, R. Tammaro, and V. Marigo, "Apoptosis in retinal degeneration involves cross-talk between apoptosis-inducing factor (AIF) and caspase-12 and is blocked by calpain inhibitors," Proceedings of the National Academy of Sciences of the United States of America, vol. 103, no. 46, pp. 17366-17371, 2006.

[21] S. E. Pearce-Kelling, T. S. Aleman, A. Nickle et al., "Calcium channel blocker D-cis-diltiazem does not slow retinal degeneration in the PDE6B mutant rcd 1 canine model of pigmentosa," Molecular Vision, vol. 7, pp. 42-47, 2001.

[22] S. Mizukoshi, M. Nakazawa, K. Sato, T. Ozaki, T. Metoki, and S. I. Ishiguro, "Activation of mitochondrial calpain and release of apoptosis-indusing factor from mitochondria in RCS rat retinal degeneration," Experimental Eye Research, vol. 91, no. 3, pp. 353-361, 2010.

[23] N. Sanvicens, V. Gómez-Vicente, I. Masip, A. Messeguer, and T. G. Cotter, "Oxidative stress-induced apoptosis in retinal photoreceptor cells is mediated by calpains and caspases and blocked by the oxygen radical scavenger CR-6," Journal of Biological Chemistry, vol. 279, no. 38, pp. 39268-39278, 2004.

[24] V. Gómez-Vicente, M. Donovan, and T. G. Cotter, "Multiple death pathways in retina-derived $661 \mathrm{~W}$ cells following growth factor deprivation: crosstalk between caspases and calpains," Cell Death and Differentiation, vol. 12, no. 7, pp. 796-804, 2005.

[25] P. Nicotera and S. Orrenius, "The role of calcium in apoptosis," Cell Calcium, vol. 23, no. 2-3, pp. 173-180, 1998.

[26] D. A. Fox, A. T. Poblenz, and L. He, "Calcium overload triggers rod photoreceptor apoptotic cell death in chemical-induced and inherited retinal degenerations," Annals of the New York Academy of Sciences, vol. 893, pp. 282-285, 1999.

[27] M. N. Delyfer, T. Léveillard, S. Mohand-Saïd, D. Hicks, S. Picaud, and J. A. Sahel, "Inherited retinal degenerations: therapeutic prospects," Biology of the Cell, vol. 96, no. 4, pp. 261-269, 2004.

[28] F. Paquet-Durand, L. Johnson, and P. Ekström, "Calpain activity in retinal degeneration," Journal of Neuroscience Research, vol. 85, no. 4, pp. 693-702, 2007.

[29] F. Doonan, M. Donovan, and T. G. Cotter, "Activation of multiple pathways during photoreceptor apoptosis in the rd mouse," Investigative Ophthalmology and Visual Science, vol. 46, no. 10, pp. 3530-3538, 2005.

[30] E. C. Steele Jr., X. Chen, P. M. Iuvone, and P. R. MacLeish, "Imaging of $\mathrm{Ca} 2+$ dynamics within the presynaptic terminals of salamander rod photoreceptors," Journal of Neurophysiology, vol. 94, no. 6, pp. 4544-4553, 2005.

[31] T. Szikra and D. Krizaj, "Calcium signals in inner segments of photoreceptors," in The Visual Transduction Cascade: Basic and Clinical Principles, J. Tombran-Tink and C. Barnstable, Eds., pp. 197-223, Jumana Press, Totowa, NJ, USA, 2009.
[32] P. Barabas, C. C. Peck, and D. Krizaj, "Do calcium channel blockers rescue dying photoreceptors in the Pde6b $b^{r d 1}$ mouse?" Advances in Experimental Medicine and Biology, vol. 664, pp. 491-499, 2010.

[33] S. A. Susin, H. K. Lorenzo, N. Zamzami et al., "Molecular characterization of mitochodrial apoptosis-inducing factor," Nature, vol. 397, no. 6718, pp. 441-446, 1999.

[34] P. Widlak, L. Y. Li, X. Wang, and W. T. Garrard, "Action of recombinant human apoptotic endonuclease $G$ on naked DNA and chromatin substrates: cooperation with exonuclease and DNase I," Journal of Biological Chemistry, vol. 276, no. 51, pp. 48404-48409, 2001.

[35] M. Frasson, J. A. Sahel, M. Fabre, M. Simonutti, H. Dreyfus, and S. Picaud, "Retinitis pigmentosa: rod photoreceptor rescue by a calcium-channel blocker in the rd mouse," Nature Medicine, vol. 5, no. 10, pp. 1183-1187, 1999.

[36] B. S. Pawlyk, T. Li, M. S. Scimeca, M. A. Sandberg, and E. L. Berson, "Absence of photoreceptor rescue with D-cisdiltiazem in the rd mouse," Investigative Ophthalmology and Visual Science, vol. 43, no. 6, pp. 1912-1915, 2002.

[37] H. Yamazaki, H. Ohguro, T. Maeda et al., "Preservation of retinal morphology and functions in Royal College Surgeons rat by nilvadipine, a Ca2+ Antagonist," Investigative Ophthalmology and Visual Science, vol. 43, no. 4, pp. 919-926, 2002.

[38] M. Donovan and T. G. Cotter, "Caspase-independent photoreceptor apoptosis in vivo and diffrential expression of apoptotic protease activating factor-1 and caspase-3 during retinal development," Cell Death and Differentiation, vol. 9, no. 11, pp. 1220-1231, 2002.

[39] J. Hart, M. F. Wilkinson, M. E. M. Kelly, and S. Barnes, "Inhibitory action of diltiazem on voltage-gated calcium channels in cone photoreceptors," Experimental Eye Research, vol. 76, no. 5, pp. 597-604, 2003.

[40] M. Sato, H. Ohguro, I. Ohguro et al., "Study of pharmacological effects of nilvadipine on RCS rat retinal degeneration by microarray analysis," Biochemical and Biophysical Research Communications, vol. 306, no. 4, pp. 826-831, 2003.

[41] Y. Takano, H. Ohguro, M. Dezawa et al., "Study of drug effects of calcium channel blockers on retinal degeneration of rd mouse," Biochemical and Biophysical Research Communications, vol. 313, no. 4, pp. 1015-1022, 2004.

[42] R. A. Bush, L. Kononen, S. Machida, and P. A. Sieving, "The effect of calcium channel blocker diltiazem on photoreceptor degeneration in the rhodopsin Pro23His rat," Investigative Ophthalmology and Visual Science, vol. 41, no. 9, pp. 26972701, 2000.

[43] K. Takeuchi, M. Nakazawa, and S. Mizukoshi, "Systemic administration of nilvadipine delays photoreceptor degeneration of heterozygous retinal degeneration slow (rds) mouse," Experimental Eye Research, vol. 86, no. 1, pp. 60-69, 2008.

[44] K. W. Koch and U. B. Kaupp, "Cyclic GMP directly regulates a cation conductance in membranes of bovine rods by a cooperative mechanism," Journal of Biological Chemistry, vol. 260, no. 11, pp. 6788-6800, 1985.

[45] D. Cia, A. Bordais, C. Varela et al., "Voltage-gated channels and calcium homeostasis in mammalian rod photoreceptors," Journal of Neurophysiology, vol. 93, no. 3, pp. 1468-1475, 2005.

[46] D. S. Read, M. A. McCall, and R. G. Gregg, "Absence of voltage-dependent calcium channels delays photoreceptor degeneration in rd mice," Experimental Eye Research, vol. 75, no. 4, pp. 415-420, 2002.

[47] Y. Tokuma, T. Fujiwara, and H. Noguchi, "Absorption, distribution and excretion of nilvadipine, a new dihydropyridine 
calcium antagonist, in rats and dogs," Xenobiotica, vol. 17, no. 11, pp. 1341-1349, 1987.

[48] N. Koseki, M. Araie, A. Tomidokoro et al., "A placebocontrolled 3-year study of a calcium blocker on visual field and ocular circulation in glaucoma with low-normal pressure," Ophthalmology, vol. 115, no. 11, pp. 2049-2057, 2008.

[49] E. G. Faktorovich, R. H. Steinberg, D. Yasumura, M. T. Matthes, and M. M. LaVail, "Photoreceptor degeneration in inherited retinal dystrophy delayed by basic fibroblast growth factor," Nature, vol. 347, no. 6288, pp. 83-86, 1990.

[50] M. Cayouette, D. Behn, M. Sendtner, P. Lachapelle, and C. Gravel, "Intraocular gene transfer of ciliary neurotrophic factor prevents death and increases responsiveness of rod photoreceptors in the retinal degeneration slow mouse," Journal of Neuroscience, vol. 18, no. 22, pp. 9282-9293, 1998.

[51] F. Q. Liang, N. S. Dejneka, D. R. Cohen et al., "AAVmediated delivery of ciliary neurotrophic factor prolongs photoreceptor survival in the rhodopsin knockout mouse," Molecular Therapy, vol. 3, no. 2, pp. 241-248, 2001.

[52] F. Q. Liang, T. S. Aleman, N. S. Dejneka et al., "Long-term protection of retinal structure but not function using rAAV.CNTF in animal models of retinitis pigmentosa," Molecular Therapy, vol. 4, no. 5, pp. 461-472, 2001.

[53] W. Tao, R. Wen, M. B. Goddard et al., "Encapsulated cellbased delivery of CNTF reduces photoreceptor degeneration in animal models of retinitis pigmentosa," Investigative Ophthalmology and Visual Science, vol. 43, no. 10, pp. 3292-3298, 2002.

[54] D. Bok, D. Yasumura, M. T. Matthes et al., "Effects of adenoassociated virus-vectored ciliary neurotrophic factor on retinal structure and function in mice with a P216L rds/peripherin mutation," Experimental Eye Research, vol. 74, no. 6, pp. 719$735,2002$.

[55] F. C. Schlichtenbrede, A. MacNeil, J. W. B. Bainbridge et al., "Intraocular gene delivery of ciliary neurotrophic factor results in significant loss of retinal function in normal mice and in the Prph2 model of retinal degeneration," Gene Therapy, vol. 10, no. 6, pp. 523-527, 2003.

[56] R. Wen, Y. Song, S. Kjellstrom et al., "Regulation of rod phototransduction machinery by ciliary neurotrophic factor," Journal of Neuroscience, vol. 26, no. 52, pp. 13523-13530, 2006.

[57] P. A. Sieving, R. C. Caruso, W. Tao et al., "Ciliary neurotrophic factor (CNTF) for human retinal degeneration: phase I trial of CNTF delivered by encapsulated cell intraocular implants," Proceedings of the National Academy of Sciences of the United States of America, vol. 103, no. 10, pp. 3896-3901, 2006.

[58] R. J. Carmody and T. G. Cotter, "Oxidative stress induces caspase-independent retinal apoptosis in vitro," Cell Death and Differentiation, vol. 7, no. 3, pp. 282-291, 2000.

[59] D. Y. Yu, S. Cringle, K. Valter, N. Walsh, D. Lee, and J. Stone, "Photoreceptor death, trophic factor expression, retinal oxygen status, and photoreceptor function in the $\mathrm{P} 23 \mathrm{H}$ rat," Investigative Ophthalmology and Visual Science, vol. 45, no. 6, pp. 2013-2019, 2004.

[60] J. Shen, X. Yang, A. Dong et al., "Oxidative damage is a potential cause of cone cell death in retinitis pigmentosa," Journal of Cellular Physiology, vol. 203, no. 3, pp. 457-464, 2005.

[61] K. Komeima, B. S. Rogers, L. Lu, and P. A. Campochiaro, "Antioxidants reduce cone cell death in a model of retinitis pigmentosa," Proceedings of the National Academy of Sciences of the United States of America, vol. 103, no. 30, pp. 11300-11305, 2006.
[62] M. Tuson, A. Garanto, R. Gonzàlez-Duarte, and G. Marfany, "Overexpression of CERKL, a gene responsible for retinitis pigmentosa in humans, protects cells from apoptosis induced by oxidative stress," Molecular Vision, vol. 15, pp. 168-180, 2009.

[63] S. Usui, K. Komeima, S. Y. Lee et al., "Increased expression of catalase and superoxide dismutase 2 reduces cone cell death in retinitis pigmentosa," Molecular Therapy, vol. 17, no. 5, pp. 778-786, 2009.

[64] H. Sugawara, K. Tobise, and K. Kikuchi, "Antioxidant effects of calcium antagonists on rat myocardial membrane lipid peroxidation," Hypertension Research, vol. 19, no. 4, pp. 223228, 1996.

[65] H. Pasantes-Morales, H. Quiroz, and O. Quesada, “Treatment with taurine, diltiazem, and vitamin E retards the progressive visual field reduction in retinitis pigmentosa: a 3-year followup study," Metabolic Brain Disease, vol. 17, no. 3, pp. 183-197, 2002.

[66] H. Ohguro, "New drug therapy for retinal degeneration," Nippon Ganka Gakkai zasshi, vol. 112, no. 1, pp. 7-21, 2008.

[67] M. Nakazawa, H. Ohguro, K. Takeuchi, Y. Miyagawa, T. Ito, and T. Metoki, "Effect of nilvadipine on central visual field in retinitis pigmentosa: a 30-month clinical trial," Ophthalmologica, vol. 225, no. 2, pp. 120-126, 2010.

[68] E. L. Berson, B. Rosner, M. A. Sandberg et al., "A randomized trial of vitamin A and vitamin E supplementation for retinitis pigmentosa," Archives of Ophthalmology, vol. 111, no. 6, pp. 761-772, 1993.

[69] E. L. Berson, "Long-term visual prognoses in patients with retinitis pigmentosa: the Ludwig von Sallmann lecture," Experimental Eye Research, vol. 85, no. 1, pp. 7-14, 2007.

[70] E. L. Berson, B. Rosner, M. A. Sandberg et al., "Clinical trial of lutein in patients with retinitis pigmentosa receiving vitamin A," Archives of Ophthalmology, vol. 128, no. 4, pp. 403-411, 2010.

[71] G. A. Fishman, L. D. Gilbert, R. G. Fiscella, A. E. Kimura, and L. M. Jampol, "Acetazolamide for treatment of chronic macular edema in retinitis pigmentosa," Archives of Ophthalmology, vol. 107, no. 10, pp. 1445-1452, 1989.

[72] S. Grover, G. A. Fishman, R. G. Fiscella, and A. E. Adelman, "Efficacy of dorzolamide hydrochloride in the management of chronic cystoid macular edema in patients with retinitis pigmentosa," Retina, vol. 17, no. 3, pp. 222-231, 1997.

[73] M. Araie and C. Yamaya, "Use of calcium channel blockers for glaucoma," Progress in Retinal and Eye Research. In press. 


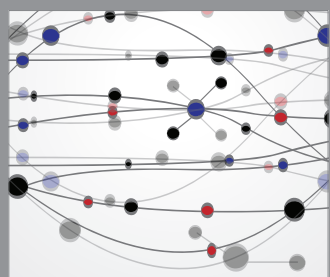

The Scientific World Journal
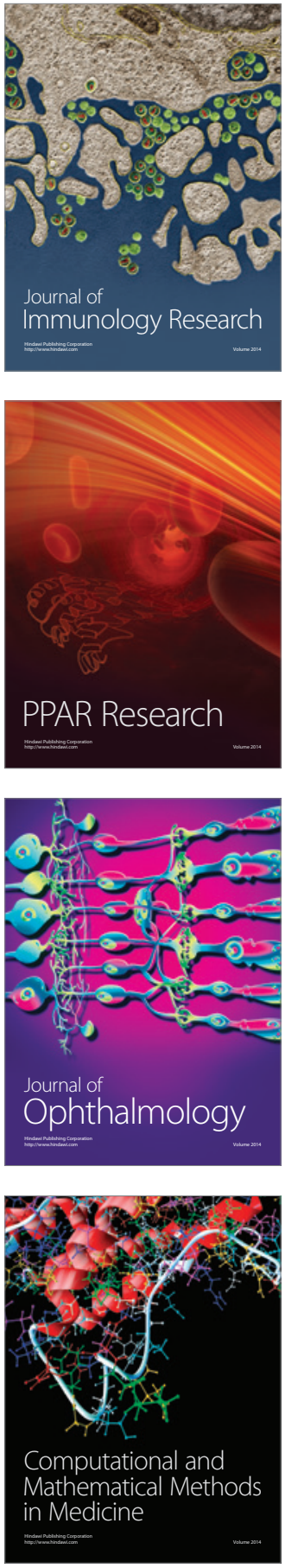

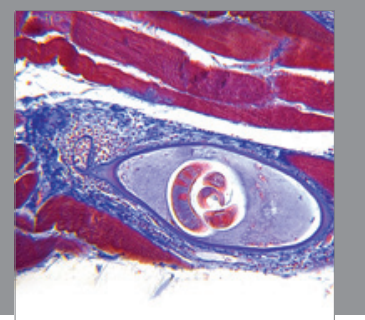

Gastroenterology

Research and Practice
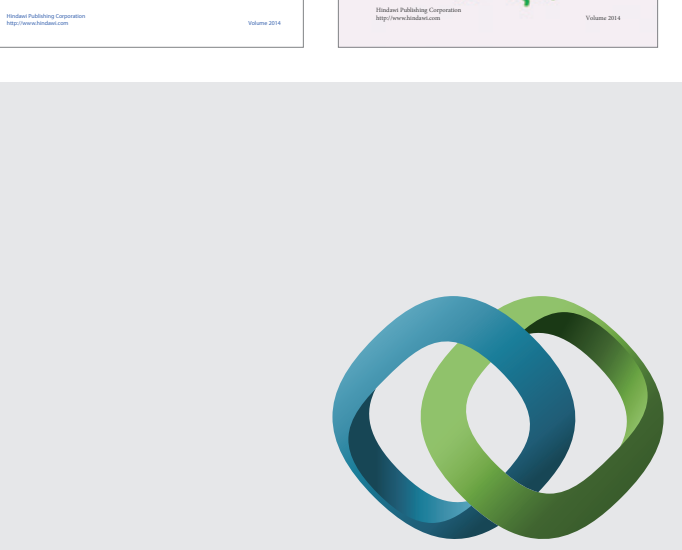

\section{Hindawi}

Submit your manuscripts at

http://www.hindawi.com
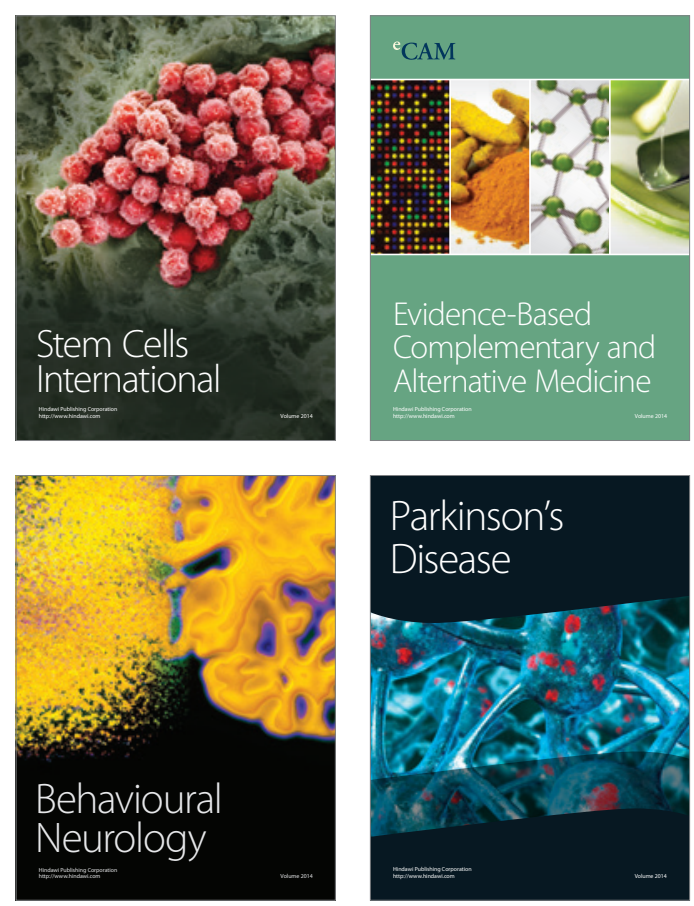

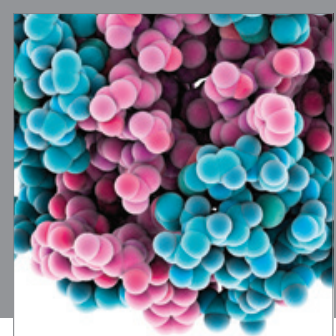

Journal of
Diabetes Research

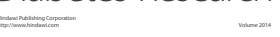

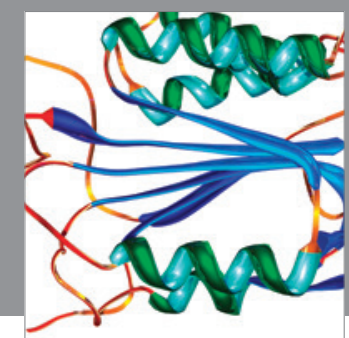

Disease Markers
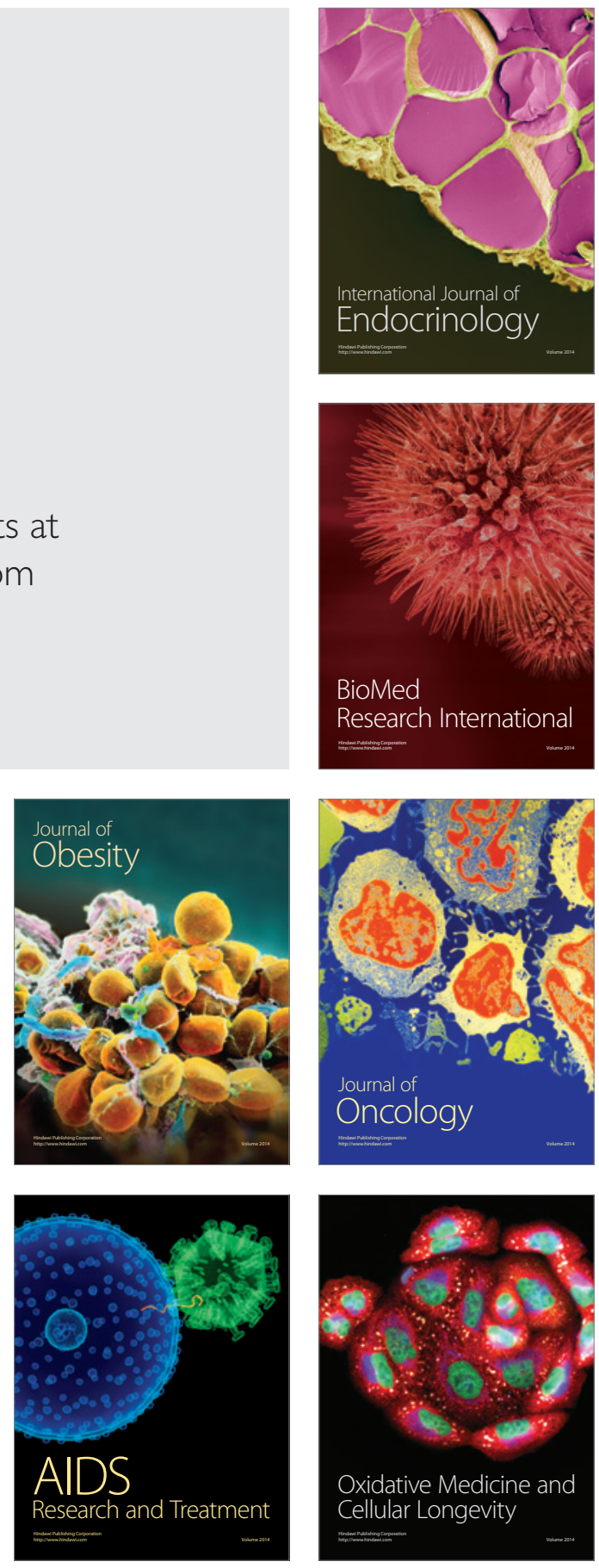